\title{
Comparación del Método del Principio de la Máxima Entropía en la Estimación de Parámetros de la Distribución de Valores Extremos Tipo I
}

\section{José A. Raynal}

Universidad de las Américas-Puebla, Departamento de Ingeniería Civil y Ambiental, Santa Catarina Mártir, 72820 Cholula, Puebla-México (e-mail: josea.raynal@udlap.mx)

\begin{abstract}
Resumen
Se analiza el método del Principio de la Máxima Entropía (PME) para la estimación de los parámetros de la distribución de valores extremos tipo I (VEI). El método PME ha sido comparado con otros de uso común, como son los de momentos (MOM), máxima verosimilitud (MV) y momentos de probabilidad pesada (MPP), tanto con datos reales, como por medio de experimentos de muestreo distribucional. El método PME resultó ser una opción viable adicional para estimar los parámetros de la distribución VEI, aunque no tan buena como la de los métodos de MPP, MV y MOM. También se detectó que el método PME funciona mejor cuando la muestra de datos es mayor a 50 valores de caudales máximo anuales.
\end{abstract}

Palabras clave: máxima entropía, análisis de frecuencias, caudales máximos anuales, estimación de parámetros, valores extremos

\section{Comparison of the Method of the Principle of Maximum Entropy for the Estimation of Parameters of the Extreme Value Type I Distribution}

\begin{abstract}
The method of the Principle of Maximum Entropy (POME) applied to the estimation of parameters of the extreme value type I distribution, (EVI) is analyzed. The POME method has been compared with others of widespread use, like the methods of moments (MOM), maximum likelihood (ML) and probability weighted moments (PWM), with both real flood data and through distributional sampling experiments. The POME method was another good option for estimating the parameters of the EVI distribution, but not as good as those provided by the methods of PWM, ML and MOM. It was also detected that the POME method has a better performance when the sample size is bigger than 50 values of maximum annual floods.
\end{abstract}

Keywords: maximum entropy, frequency analysis, floods, parameter estimation, extreme values 


\section{INTRODUCCIÓN}

La selección de la función de distribución de probabilidad y la elección del mejor método para estimar sus parámetros, han sido siempre asuntos de gran preocupación en el ámbito de la hidrología superficial. La literatura técnica sobre análisis de caudales máximos anuales usando la distribución de valores extremos tipo I (VEI), es abundante (Singh, 1998; Rao y Hamed, 2000).

A la fecha han sido propuestos varios métodos de estimación de parámetros para la distribución VEl, los más tradicionales son: momentos (M) y máxima verosimilitud (MV). Recientemente, se han propuesto métodos más novedosos como el de momentos de probabilidad pesada (MPP) (Greenwood et al., 1979) y el del principio de máxima entropía (PME) (Jaynes, 1961 y 1982). Es conveniente decir que existen varios métodos más que se han propuesto para el mismo fin, pero ninguno de ellos ha demostrado ser superior a los antes descritos (Raynal y Salas, 1986; Jain y Singh, 1986; Phien, 1987). En particular, se ha encontrado que los métodos de mínimos cuadrados, de rango intercuartil de la moda, momentos mixtos y medias incompletas son muy ineficientes en la estimación de los parámetros de la distribución VEI (Raynal y Salas, 1986; Jain y Singh, 1986; Phien, 1987).

En fechas recientes, el método PME ha sido escasamente aplicado en la estimación de parámetros de la distribución VEI en el campo de la hidrología superficial, en otras áreas se han presentado aplicaciones del principio de máxima entropía en la modelación física regional de crecientes (SolanaOrtega y Solana, 2001), en la representación de la lluvia intermitente (Koutsoyiannis, 2006), para la obtención de relaciones geométricas hidráulicas (Singh et al., 2003), para hacer análisis regional de precipitación (Escalante y Domínguez, 2001) y para hacer una comparación entre los Momentos-L y los Momentos-C (Ulrych et al., 2000).

El objetivo de este artículo consiste en determinar la bondad de aplicación del método PME en el análisis de caudales máximos anuales, tanto a nivel de aplicación práctica como a nivel de bondad estadística, por medio de experimentos de muestro distribucional.

\section{METODOLOGÍA}

Función de Distribución de Probabilidad de Valores Extremos Tipo I para Máximos

La función de distribución de probabilidad de valores extremos tipo I, (VEI), es (NERC, 1975):

$F(x)=\exp \left\{-\exp \left[-\frac{\left(x-x_{0}\right)}{\alpha}\right]\right\}$

donde $\alpha, y x_{0}$ son los parámetros de escala, y ubicación, respectivamente.

La función de densidad de probabilidad está dada por (NERC, 1975):

$f(x)=\frac{1}{\alpha} \exp \left\{-\exp \left[-\frac{\left(x-x_{0}\right)}{\alpha}\right]\right\} \exp \left[-\frac{\left(x-x_{0}\right)}{\alpha}\right]$

\section{El Método del Principio de la Máxima Entropía}

El método del Principio de la Máxima Entropía (PME) ha sido definido y aplicado a varias funciones de distribución de probabilidad, con funciones de densidad de probabilidad definidas y directamente 
invertibles (Singh, 1998). El método del PME aplicado a la estimación de parámetros de funciones de distribución probabilidad, consiste en los siguientes pasos principales (Singh, 1998):

1) Definir la información dada en términos de las restricciones

2) Maximizar la entropía sujeta a la información dada

3) Relacionar los parámetros a la información dada

El método PME fue formulado por Jaynes $(1961,1982)$. Este principio establece que "la asignación de probabilidades con un grado de prejuicio mínimo es aquella que maximiza la entropía sujeta a la información dada".

Estimadores de Máxima Entropía de los Parámetros de la Distribución de Valores Extremos Tipo I

Al tomar el logaritmo natural de la ecuación (2), se tiene que:

$\operatorname{Ln}[f(x)]=-\operatorname{Ln}(\alpha)-\exp \left[-\frac{\left(x-x_{0}\right)}{\alpha}\right]-\left(\frac{x-x_{0}}{\alpha}\right)$

y la entropía funcional de Shannon (EFS) para la función de distribución VEI es (Singh, 1998):

$I[f]=-\frac{1}{\alpha} \int_{-\infty}^{\infty}\left\{\exp \left[-\exp \left(-\frac{\left(x-x_{0}\right)}{\alpha}\right)\right] \exp \left(-\frac{\left(x-x_{0}\right)}{\alpha}\right)\left[-\operatorname{Ln}(\alpha)-\exp \left[-\frac{\left(x-x_{0}\right)}{\alpha}\right]-\left(\frac{\left(x-x_{0}\right)}{\alpha}\right)\right]\right\} d x(4)$

De aquí, se tiene que las restricciones apropiadas para la ecuación (2) son (Singh, 1998):

$\int_{-\infty}^{\infty} f(x) d x=1$

$\int_{-\infty}^{\infty} x f(x) d x=\bar{x}$

$\int_{-\infty}^{\infty} \exp \left(-\frac{x}{\alpha}\right) f(x) d x=E\left[\exp \left(-\frac{x}{\alpha}\right)\right]$

La función de densidad de probabilidad menos sesgada, basada en el PME, y que es consistente con las ecuaciones (5)-(7), tiene la siguiente forma (Singh, 1998):

$$
f(x)=\exp \left(-\lambda_{0}-\lambda_{1} x-\lambda_{2} \exp \left(-\frac{x}{\alpha}\right)\right)
$$

El multiplicador de Lagrange de orden cero es (Singh, 1998): 
$\lambda_{0}=\operatorname{Ln}\left[\int_{-\infty}^{\infty} \exp \left(-\lambda_{1} x-\lambda_{2} \exp \left(-\frac{x}{\alpha}\right)\right) d x\right]$

donde $\lambda_{1}$ y $\lambda_{2}$ son los multiplicadores de Lagrange de orden uno y dos, respectivamente.

También, $\lambda_{0}$ puede expresarse como (Singh, 1998):

$\lambda_{0}=\operatorname{Ln}(\alpha)-\alpha \lambda_{1} \operatorname{Ln}\left(\lambda_{2}\right)+\operatorname{Ln}\left[\Gamma\left(\alpha \lambda_{1}\right)\right]$

donde $\Gamma($.$) es la función Gamma completa de (.). Derivando las ecuaciones (9) y (10) con respecto a$ $\lambda_{1}$ y $\lambda_{2}$, se obtiene lo siguiente:

$\frac{\partial \lambda_{0}}{\partial \lambda_{1}}=-\alpha \operatorname{Ln}\left(\lambda_{2}\right)+\frac{\partial}{\partial \lambda_{1}}\left[\operatorname{Ln}\left[\Gamma\left(\alpha \lambda_{1}\right)\right]\right]$

$\frac{\partial \lambda_{0}}{\partial \lambda_{2}}=-\frac{\alpha \lambda_{1}}{\lambda_{2}}$

$\frac{\partial \lambda_{0}}{\partial \lambda_{1}}=-\bar{x}$

$\frac{\partial \lambda_{0}}{\partial \lambda_{2}}=-E\left[\exp \left(-\frac{x}{\alpha}\right)\right]$

La relación entre los parámetros y las restricciones puede construirse al sustituir la ecuación (9) en la ecuación (8) (Singh, 1998):

$$
f(x)=\frac{\left(\lambda_{2}\right)^{\alpha \lambda_{1}}}{\alpha \Gamma\left(\alpha \lambda_{1}\right)} \exp \left(-\lambda_{1} x-\lambda_{2} \exp \left(-\frac{x}{\alpha}\right)\right)
$$

y por comparación con la ecuación (2) se tiene que:

$\lambda_{1}=\alpha$

y: 
$\lambda_{2}=\exp \left(\frac{x_{0}}{\alpha}\right)$

La relación entre los parámetros y las restricciones se puede establecer como:

$\bar{x}=x_{0}+0.5772 \alpha$

$E\left[\exp \left(-\frac{\left(x-x_{0}\right.}{\alpha}\right)\right]=1$

Finalmente, las expresiones necesarias para estimar los parámetros de la distribución VEI son (Jowitt, 1979; Jain y Singh, 1987):

$$
\begin{gathered}
x_{0}=\bar{x}-0.5772 \alpha \\
E\left[\exp \left(-\frac{\left(x-x_{0}\right)}{\alpha}\right)\right]=1
\end{gathered}
$$

Un estimador muestral de la ecuación (21) es (Jowitt, 1979):

$$
\frac{1}{N} \sum_{i=1}^{N} \exp \left(-\frac{\left(x_{i}-x_{0}\right)}{\alpha}\right)=1
$$

donde $\bar{x}$ es la media de los datos y $\mathrm{N}$ es el tamaño de muestra.

El procedimiento antes descrito implica hacer una búsqueda de raíces de la ecuación (22), proponiendo primero un estimador del parámetro de escala, $\alpha$, encontrar el valor del parámetro de ubicación, $x_{0}$, por medio de la ecuación (20) e ir ajustando ambos parámetros con cualquier técnica de búsqueda de raíces. El método PME es particularmente adaptable para ser estimado por medio de una hoja de cálculo, como por ejemplo Excel ${ }^{\circledR}$ (Marca Registrada de Microsoft, Inc.).

\section{RESULTADOS Y DISCUSIÓN}

\section{Aplicación a Muestras de Caudales Reales}

Como ejemplos de aplicación, los datos de caudales máximos anuales de 10 estaciones hidrométricas, ubicadas en las Regiones Hidrológicas números 9 y 10 de los estados de Sonora y Sinaloa, en el Noroeste de México, ver figura 1, fueron procesados y calculados los estimadores producidos por los métodos PME, MV, MOM y MPP para los parámetros de la distribución VEI, para dichas muestras de datos; así como, sus errores estándar de ajuste y las desviaciones absolutas promedio. Los detalles de los estimadores MV, MOM y MPP pueden encontrarse en Rao y Hamed (2000). El área drenada, media, desviación estándar y el coeficiente de asimetría de los datos de muestra de las estaciones citadas, se indican en la tabla 1. 


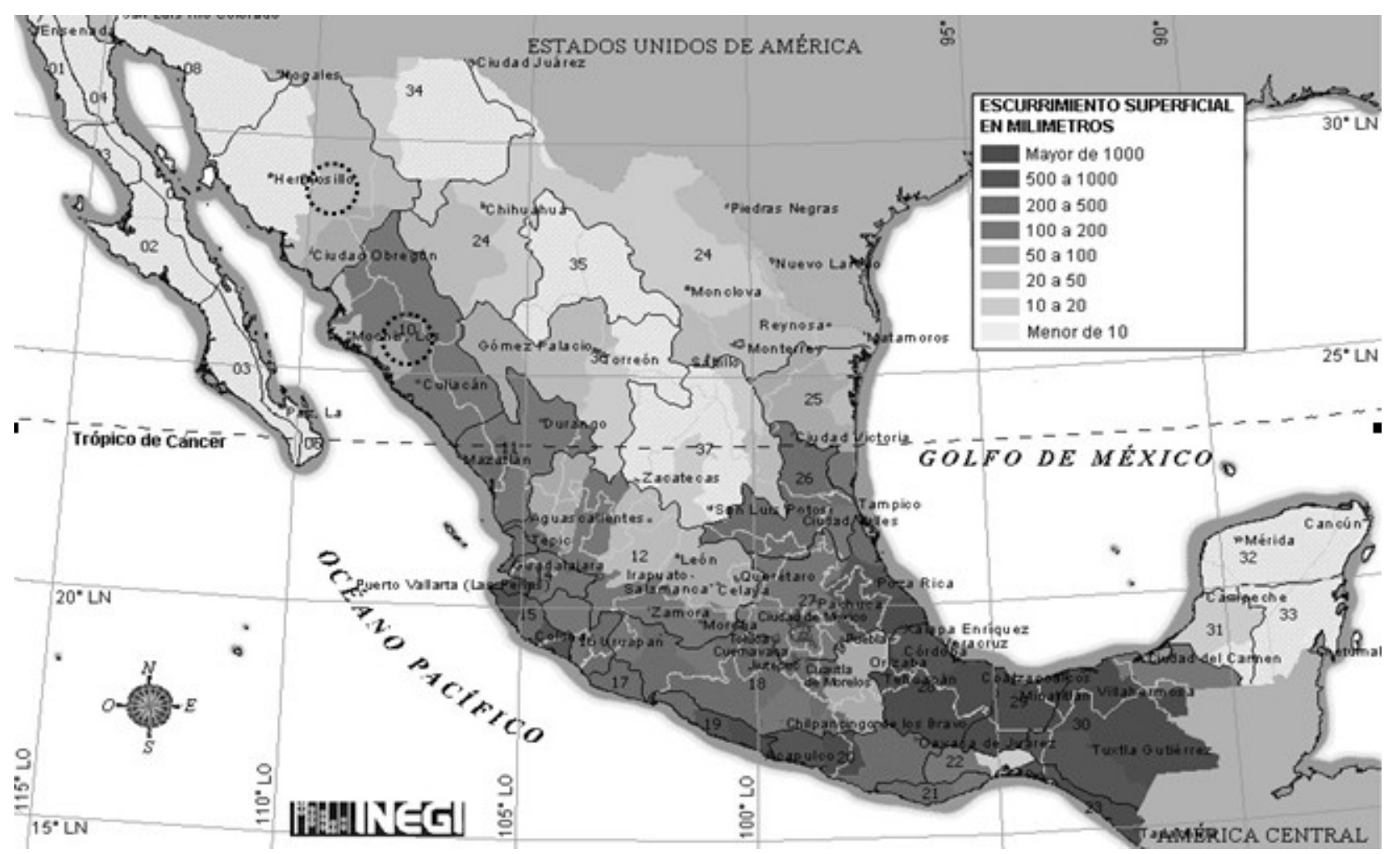

Fig. 1: Regiones Hidrológicas de México

Tabla 1: Datos estadísticos de las estaciones hidrométricas seleccionadas

\begin{tabular}{|c|c|c|c|c|c|c|c|}
\hline $\begin{array}{c}\text { Área de } \\
\text { Cuenca } \\
\left(\mathrm{km}^{2}\right)\end{array}$ & $\begin{array}{l}\text { Media } \\
\left(\mathrm{m}^{3} / \mathrm{s}\right)\end{array}$ & $\begin{array}{c}\text { Desviación } \\
\text { Estándar } \\
\left(\mathrm{m}^{3} / \mathrm{s}\right)\end{array}$ & $\begin{array}{c}\text { Coeficiente } \\
\text { de } \\
\text { Asimetría }\end{array}$ & $\begin{array}{c}\text { Área de } \\
\text { Cuenca } \\
\left(\mathrm{km}^{2}\right)\end{array}$ & $\begin{array}{l}\text { Media } \\
\left(\mathrm{m}^{3} / \mathrm{s}\right)\end{array}$ & $\begin{array}{c}\text { Desviación } \\
\text { Estándar } \\
\left(\mathrm{m}^{3} / \mathrm{s}\right)\end{array}$ & $\begin{array}{c}\text { Coeficiente } \\
\text { de } \\
\text { Asimetría }\end{array}$ \\
\hline \multicolumn{4}{|c|}{ Jaina } & \multicolumn{4}{|c|}{ Ixpalino } \\
\hline 8179 & 1095.9 & 1163.7 & 3.4 & 6166 & 864.9 & 808.1 & 2.8 \\
\hline \multicolumn{4}{|c|}{ Santa Cruz } & \multicolumn{4}{|c|}{ Acatitán } \\
\hline 8919 & 1223.4 & 1204.0 & 3.1 & 1884 & 410.5 & 464.0 & 2.7 \\
\hline \multicolumn{4}{|c|}{ El Orégano } & \multicolumn{4}{|c|}{ San Bernardo } \\
\hline 11606 & 153.7 & 96.3 & 0.7 & 7510 & 741.6 & 550.2 & 2.1 \\
\hline \multicolumn{4}{|c|}{ Huites } & \multicolumn{4}{|c|}{ Choix } \\
\hline 26020 & 2499.0 & 2221.4 & 2.1 & 1403 & 160.7 & 112.7 & 2.5 \\
\hline \multicolumn{4}{|c|}{ El Zopilote } & \multicolumn{4}{|c|}{ Tezocoma } \\
\hline 666 & 163.4 & 167.6 & 2.1 & 901 & 87.8 & 125.1 & 3.8 \\
\hline
\end{tabular}

Para medir la bondad del ajuste por varios métodos se calculó el error estándar de ajuste, EE, dado por Kite (1988):

$E E=\left[\frac{\sum_{i=1}^{N}\left(x_{i}-y_{i}\right)^{2}}{\left(N-m_{j}\right)}\right]^{1 / 2}$ 
donde $x_{i}$ son los valores históricos de la muestra de datos, $y_{i}$ son los valores producidos por la función de distribución correspondiente a los periodos de retorno de los valores históricos, $\mathrm{N}$ es el tamaño de la muestra, y m $\mathrm{m}_{\mathrm{j}}$ es el número de parámetros de la función de distribución.

Se calculó también otra medida común de bondad de ajuste conocida como la desviación absoluta relativa promedio, DARP, cuya formulación es como sigue (Jain y Singh, 1987):

$D A R P=\frac{100}{N} \sum_{i=1}^{N}\left|\frac{\left(x_{i}-y_{i}\right)}{x_{i}}\right|$

donde $x_{i}, y_{i}$ y $\mathrm{N}$ fueron definidas previamente.

Los parámetros de la distribución VEI, obtenidos por los métodos del principio de máxima entropía, máxima verosimilitud, momentos y momentos de probabilidad pesada, para las diez estaciones seleccionadas se muestran en la tabla 2. En esta tabla se muestran también los EE y las DARP para dichos ajustes.

Tabla 2: Parámetros, errores estándar de ajuste y desviaciones absolutas promedio de la distribución VEI de las diez estaciones hidrométricas seleccionadas

\begin{tabular}{|c|c|c|c|c|c|c|c|c|c|}
\hline Método & \multicolumn{2}{|c|}{ Parámetros } & EE & MDAR & Método & \multicolumn{2}{|c|}{ Parámetros } & EE & MDAR \\
\hline & Ubicación & Escala & & & & Ubicaciór & Escala & & \\
\hline \multicolumn{5}{|c|}{ Jaina } & \multicolumn{5}{|c|}{ Ixpalino } \\
\hline PME & 464.08 & 402.64 & 345 & 0.63 & PME & 586.5 & \begin{tabular}{l|l}
482.2 \\
\end{tabular} & 425 & 58.3 \\
\hline MV & 451.58 & 361.29 & 372 & 0.95 & MV & 572.4 & 438.5 & 417 & 53.1 \\
\hline $\mathrm{M}$ & 388.13 & 534.27 & 312 & 32.17 & $\mathrm{M}$ & 505.7 & 622.3 & 380 & 45.4 \\
\hline MPP & 439.47 & 445.28 & 327 & 10.85 & MPP & 559.3 & 529.5 & 404 & 53.9 \\
\hline \multicolumn{5}{|c|}{ Santa Cruz } & \multicolumn{5}{|c|}{ Acatitán } \\
\hline PME & 596.64 & 300.31 & 409 & 28.50 & PME & 251.0 & 276.4 & 120 & 1881.4 \\
\hline MV & 511.85 & 396.32 & 341 & 0.64 & MV & 231.6 & 242.1 & 121 & 1254.3 \\
\hline $\mathrm{M}$ & 458.32 & 540.00 & 289 & 22.69 & $\mathrm{M}$ & 201.7 & 361.8 & 149 & 4705.3 \\
\hline MPP & 501.87 & 464.50 & 305 & 6.57 & MPP & 233.4 & 306.9 & 122 & 2889.5 \\
\hline \multicolumn{5}{|c|}{ El Orégano } & \multicolumn{5}{|c|}{ San Bernardo } \\
\hline PME & 109.27 & 77.03 & 14 & 0.96 & PME & 536.7 & 355.0 & 218 & 3.0 \\
\hline MV & 108.84 & 75.98 & 14 & 0.81 & $\mathrm{MV}$ & 527.2 & 325.5 & 241 & 1.4 \\
\hline $\mathrm{M}$ & 109.95 & 75.95 & 15 & 2.90 & $\mathrm{M}$ & 494. & 429.5 & 188 & 5.7 \\
\hline MPP & 107.74 & 79.68 & 12 & 1.72 & MPP & 516.2 & 390.5 & 200 & 1.6 \\
\hline \multicolumn{5}{|c|}{ Huites } & \multicolumn{5}{|c|}{ Choix } \\
\hline PME & 1698.96 & 1386.00 & 911 & 2.28 & PME & 120.2 & 70.2 & 49 & 2.7 \\
\hline MV & 1650.26 & 1212.08 & 1046 & 0.48 & MV & 118.2 & 64.4 & 53 & 1.1 \\
\hline $\mathrm{M}$ & 1499.35 & 1733.99 & 789 & 13.42 & $\mathrm{M}$ & 109.9 & 88.0 & 43 & 5.6 \\
\hline MPP & 1616.40 & 1529.04 & 839 & 4.57 & MPP & 116.2 & \begin{tabular}{l|l}
77.1 & \\
\end{tabular} & 46 & 0.7 \\
\hline \multicolumn{5}{|c|}{ El Zopilote } & \multicolumn{5}{|c|}{ Tezocoma } \\
\hline PME & 99.92 & 109.92 & 62 & 88.20 & PME & 51.1 & 63.6 & 78 & 90.1 \\
\hline $\mathrm{MV}$ & 104.53 & 102.79 & 69 & 48.34 & $\mathrm{MV}$ & 48.7 & 54.4 & 84 & 57.8 \\
\hline $\mathrm{M}$ & 87.93 & 130.70 & 54 & 199.16 & $\mathrm{M}$ & 31.5 & 97.6 & 72 & 387.4 \\
\hline MPP & 94.05 & 120.10 & 57 & 142.55 & MPP & 46.4 & 71.7 & 75 & 164.9 \\
\hline
\end{tabular}

De la información contenida en la tabla 2, se observó que el error estándar de ajuste, EE, que muestra los mejores resultados es el método MOM con ocho valores mínimos del EE de diez 
posibles. Fue seguido por los métodos de MPP y del PME, con uno cada uno. De acuerdo con la desviación absoluta relativa promedio, DARP, contenida en la tabla 2, el método que muestra los mejores resultados es el MV con siete valores mínimos del DARP de diez posibles. Fue seguido por los métodos PME, MPP y MOM, con uno cada uno.

\section{Aplicación a Muestras de caudales Sintéticos}

Adicionalmente, se realizaron experimentos de muestreo distribucional para tamaños de muestra 9 , 19, 49 y 99 y números de muestras de 11000, 5210, 2020 y 1000, respectivamente. Se usaron los métodos de MOM, MV, MPP y PME.

Los experimentos de muestreo distribucional consistieron en asignar los valores poblacionales de 10 y 1 , sin pérdida de generalidad, a los parámetros de ubicación y escala, respectivamente, de la distribución VEI. El proceso consistió de los pasos siguientes:

1) Generación de números aleatorios con distribución uniforme en el intervalo $(0,1)$

2) Utilización de estos números aleatorios como valores de la función de distribución VEI

3) Conversión a valores aleatorios con distribución VEI por medio de la inversión de la ecuación (1):

$$
x=x_{0}-\alpha \operatorname{Ln}\{-\operatorname{Ln}[F(x)]\}
$$

4) Conformación de muestras de tamaños 9, 19, 49 y 99, con los valores generados en el punto anterior

5) Estimación de los parámetros de la distribución VEI, basada en la muestras anteriores y por medio de los métodos PME, MV, MOM y MPP

6) Estimación de los parámetros estadísticos de la media, sesgo y varianza de los parámetros de la distribución VEI obtenidos en el punto anterior.

El sesgo se definió como:

$\operatorname{Sesgo}=\theta-\hat{\theta}$

donde $\theta$ es el parámetro y $\hat{\theta}$ es un estimador de tal parámetro. Los resultados están contenidos en las tablas 3 y 4.

Tabla 3: Propiedades de los estimadores de PME, MOM, ML y MPP para el parámetro de ubicación $\mathrm{X}_{0}$ suponiendo un valor poblacional: $\mathrm{X}_{0}=10.0(\alpha=1.0)$

\begin{tabular}{|c|c|c|c|c|c|c|c|}
\hline Método & $\begin{array}{l}\text { Valor } \\
\text { Medio }\end{array}$ & Sesgo & Varianza & Método & $\begin{array}{l}\text { Valor } \\
\text { Medio }\end{array}$ & Sesgo & Varianza \\
\hline \multicolumn{4}{|c|}{$\mathrm{N}=9$} & \multicolumn{4}{|c|}{$\mathrm{N}=19$} \\
\hline PME & 10.0176 & 0.0176 & 0.2574 & PME & 10.0133 & 0.0133 & 0.2227 \\
\hline MV & 10.0406 & -0.0406 & 0.1219 & MV & 10.0217 & -0.0217 & 0.0587 \\
\hline MOM & 9.9932 & 0.0068 & 0.1219 & MOM & 10.0018 & -0.0018 & 0.0598 \\
\hline MPP & 9.9974 & 0.0026 & 0.1208 & MPP & 10.0019 & -0.0019 & 0.0585 \\
\hline \multicolumn{4}{|c|}{$\mathrm{N}=49$} & \multicolumn{4}{|c|}{$\mathrm{N}=99$} \\
\hline PME & 10.0188 & 0.0188 & 0.1549 & PME & 10.0187 & -0.0187 & 0.1083 \\
\hline MV & 10.0138 & -0.0138 & 0.0221 & $\mathrm{MV}$ & 10.0038 & -0.0038 & 0.0111 \\
\hline MOM & 10.0061 & -0.0061 & 0.0231 & MOM & 9.9999 & 0.0001 & 0.0118 \\
\hline MPP & 10.0060 & -0.0060 & 0.0220 & MPP & 9.9998 & 0.0002 & 0.0112 \\
\hline
\end{tabular}


Tabla 4: Propiedades de los estimadores de PME, MOM, ML y MPP para el parámetro de escala $\alpha$, suponiendo un valor poblacional: $\alpha=1.0\left(x_{0}=10.0\right)$

\begin{tabular}{|c|c|c|c|c|c|c|c|}
\hline Método & $\begin{array}{l}\text { Valor } \\
\text { Medio }\end{array}$ & Sesgo & Varianza & Método & $\begin{array}{l}\text { Valor } \\
\text { Medio }\end{array}$ & Sesgo & Varianza \\
\hline \multicolumn{4}{|c|}{$\mathrm{N}=9$} & \multicolumn{4}{|c|}{$\mathrm{N}=19$} \\
\hline PME & 0.9413 & 0.0587 & 0.2464 & PME & 0.9677 & 0.0323 & 0.1811 \\
\hline MV & 0.9086 & 0.0914 & 0.0685 & MV & 0.9566 & 0.0434 & 0.0300 \\
\hline MOM & 1.0035 & -0.0035 & 0.1142 & MOM & 0.9971 & 0.0029 & 0.0497 \\
\hline MPP & 0.9949 & 0.0051 & 0.0981 & MPP & 0.9959 & 0.0041 & 0.0406 \\
\hline \multicolumn{4}{|c|}{$N=49$} & \multicolumn{4}{|c|}{$\mathrm{N}=99$} \\
\hline PME & 0.9924 & 0.0076 & 0.1198 & PME & 1.0024 & -0.0024 & 0.0839 \\
\hline MV & 0.9832 & 0.0168 & 0.0127 & MV & 0.9889 & 0.0111 & 0.0057 \\
\hline MOM & 1.0016 & -0.0016 & 0.0222 & MOM & 0.9992 & 0.0008 & 0.0107 \\
\hline MPP & 1.0006 & -0.0006 & 0.0171 & MPP & 0.9981 & 0.0019 & 0.0077 \\
\hline
\end{tabular}

Los resultados contenidos en las tablas 3 y 4 son consistentes con lo reportado en la literatura con experimentos de muestreo distribucional. Raynal y Salas (1986) ubican al método MPP como la mejor opción, sin haber considerado el método PME. Jain y Singh (1986) encontraron que el mejor método era el de MV. Phien (1987) ubica como mejor método el de MPP. El método MOM es el mejor al reducir el sesgo, pero sus varianzas son consistentemente más grandes que las de los métodos MV y MPP. El método MPP y el MV, son consistentemente los que producen las varianzas mínimas para los parámetros de ubicación y escala, respectivamente. Se observó también que el método PME debe ser utilizado cuando el tamaño de la muestra es mayor a 50 valores de caudales máximos, ya que para tamaños de muestra más pequeños la varianza de los parámetros es muy grande.

\section{CONCLUSIONES}

Se concluye que el método del principio de máxima entropía tiene las características estadísticas necesarias para ser usado con toda confianza en el análisis de caudales máximos anuales, siempre y cuando el tamaño de muestra sea superior a 50 datos de caudales máximos anuales, en caso contrario se tendrán varianzas elevadas en la evaluación de los parámetros de la distribución VEI. El autor recomienda el uso del método del principio de máxima entropía como una alternativa viable, cuando se requiera hacer algún análisis de caudales máximos anuales, basado en los argumentos anteriores y dada su gran facilidad computacional en la obtención de los parámetros de la distribución VEI.

\section{AGRADECIMIENTOS}

A la Universidad de las Américas, Puebla por el apoyo y las facilidades otorgadas para la realización de este artículo.

\section{NOMENCLATURA}

$\begin{array}{cl}\alpha & \text { Parámetro de escala } \\ \partial & \text { Derivada parcial } \\ \Gamma(.) & \text { Función Gamma completa de }(.) \\ \lambda_{i} & \text { Multiplicador de Lagrange } \\ \theta & \text { Parámetro } \\ \Sigma & \text { Sumatoria } \\ f & \text { Integral } \\ D A R P & \text { Desviación absoluta relativa promedio } \\ E(.) & \text { Valor esperado de }(.)\end{array}$

EE Error estándar de ajuste

exp Exponencial

$f(x) \quad$ Función de densidad de probabilidad

$F(x) \quad$ Función de distribución de probabilidad

l[f] Entropía funcional de Shannon

Ln Logaritmo natural

$N \quad$ Tamaño de muestra

$\bar{x} \quad$ Media muestral

$x_{0} \quad$ Parámetro de ubicación 


\section{REFERENCIAS}

Escalante Sandoval C.A. y Domínguez Esquivel, J.Y.; Análisis Regional de Precipitación con Base en una Distribución Bivariada Ajustada por Máxima Entropía, Ingeniería Hidráulica en México: 16 (3), 91 $102(2001)$

Greenwood, J.A., J.M. Lanwher, N.C. Matalas y J.R. Wallis; Probability Weighted Moments: Definition, and Relation to Parameters of Several Distributions Expressable in Inverse Form, Water Resources Research, 15, 1049-1054 (1979).

Jain, D. y V.P. Singh; Estimating Parameters of EV1 Distribution for Flood Frequency Analysis, Water Resources Bulletin: 23(1), 59-71 (1987)

Jaynes, E.T.; Probability Theory in Science and Engineering, Mc-Graw Hill Book Company, New York, N. Y., EUA (1961).

Jaynes, E.T.; On the Rationale of Maximum Entropy Methods, Proceedings of IEEE: 70, 939-952 (1982).

Jowitt, P. W.; The Extreme-Value Type-1 Distribution and the Principle of Maximum Entropy, Journal of Hydrology: 42, 23-38 (1979)

Kite, G. W.; Frequency y Risk Analyses in Hydrology, 187, Water Resources Publications, Littleton, Colorado, EUA (1988)

Koutsoyiannis D.: An Entropic-Stochastic Representation of Rainfall Intermittency: The Origin of Clustering and Persistence, Water Resources Research: 42 (1): Art. No. W01401 (2006)

NERC, Natural Environment Research Council. Flood Studies Report, I, Hydrologic Studies, 41-87, Whitefriars Press Ltd., Londres, Inglaterra (1975)

Phien, H.N.; A Review of Methods of Parameter Estimation for the Extreme Value Type-1 Distribution, Journal of Hydrology: 90, 251-260 (1987)

Rao, A.R. y K.H. Hamed; Flood Frequency Analysis, 229-257, CRC Press, Boca Ratón, Florida, EUA (2000).

Raynal, J.A. y J.D. Salas; Estimation Procedures for the Type-1 Extreme Value Distribution, Journal of Hydrology: 87, 315-336 (1986)

Singh, V.P.; Entropy Based Parameter Estimation in Hydrology, 108-136, Kluwer Academic Publishers, Dordrecht, Holanda (1998).

Singh V.P., C.T. Yang y Z.Q. Deng; Downstream Hydraulic Geometry Relations: 1. Theoretical Development, Water Resources Research: 39 (12): Art. No. 1337 (2003)

Solana-Ortega, A. y Solana, V.; Entropy-based inference of Simple Physical Models for Regional Flood Analysis, Stochastic Environmental Research and Risk Assessment: 15(6), 415-446 (2001)

Ulrych, T.J., D.R. Velis y A.D. Woodbury; L-moments and C-moments, Stochastic Environmental Research and Risk Assessment: 14 (1), 50-68 (2000) 